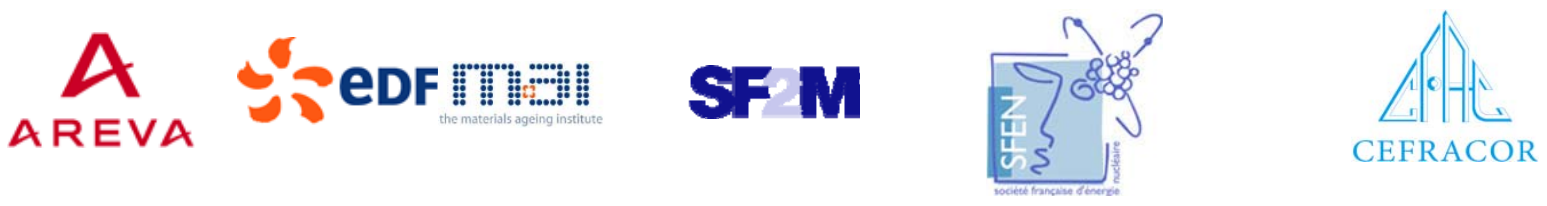

\section{MIN口S}

Centre of Excellence for Nuclear Materials

\section{Workshop}

Materials Innovation for Nuclear Optimized Systems
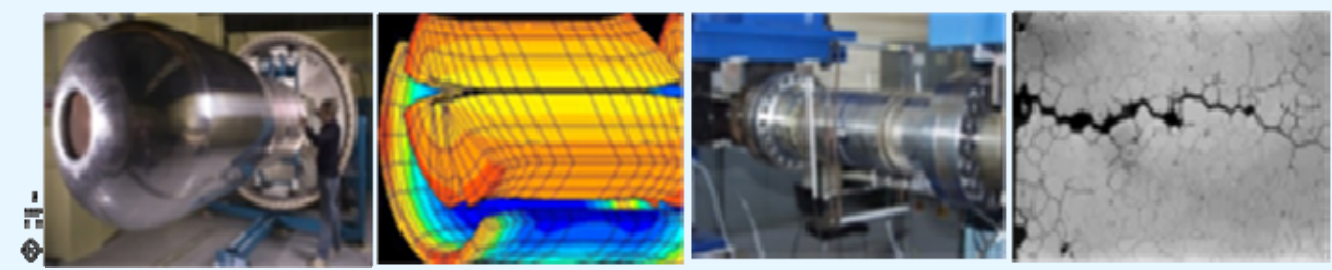

December 5-7, 2012, CEA - INSTN Saclay, France

\section{Bo SUNDMAN et al.}

KTH (Sweden) and CEA-INSTN (France)

The Use of Computational Thermodynamics to Predict Properties of Multicomponent Materials for Nuclear Applications

Workshop organized by:

Christophe GALLÉ, CEA/MINOS, Saclay - christophe.galle@cea.fr Constantin MEIS, CEA/INSTN, Saclay - constantin.meis@cea.fr 


\title{
The Use of Computational Thermodynamics to Predict Properties of Multicomponent Materials for Nuclear Applications
}

\author{
Bo SUNDMAN ${ }^{1}$, Christine GUÉNEAU ${ }^{2}$ \\ ${ }^{1} \mathrm{KTH}$ (Stockholm, Sweden), ISNTN (Saclay, France) \\ ${ }^{2}$ CEA-DEN-DPC, Service de la Corrosion et du Comportement des Matériaux dans leur Environnement, SCCME (Saclay, France)
}

Computational Thermodynamics is based on physically realistic models to describe metallic and oxide crystalline phases as well as the liquid and the gas in a consistent manner [1]. The models are used to assess experimental and theoretical data for many different materials and several thermodynamic databases has been developed for steels, ceramics, semiconductor materials as well as materials for nuclear applications.

Within CEA a long term work is ongoing to develop a database for the properties of nuclear fuels and structural materials [2].

An overview of the modeling technique will be given and several examples of the application of the database to different problems, both for traditional phase diagram calculations and its use in simulating phase transformations. The following diagrams (Fig. 1, Fig. 2 and Fig.3) show calculations in the U-Pu-O system.

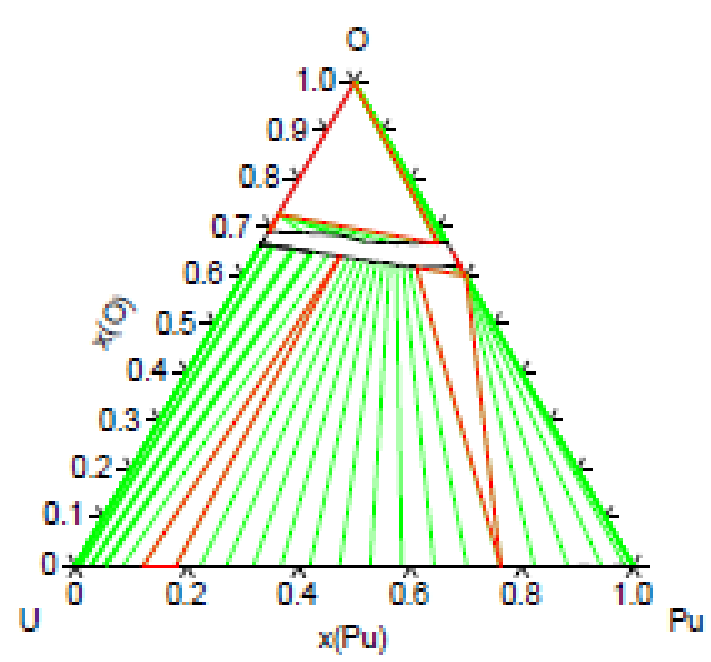

Fig. 1 : U-Pu-O phase diagram at $1273^{\circ} \mathrm{K}$.

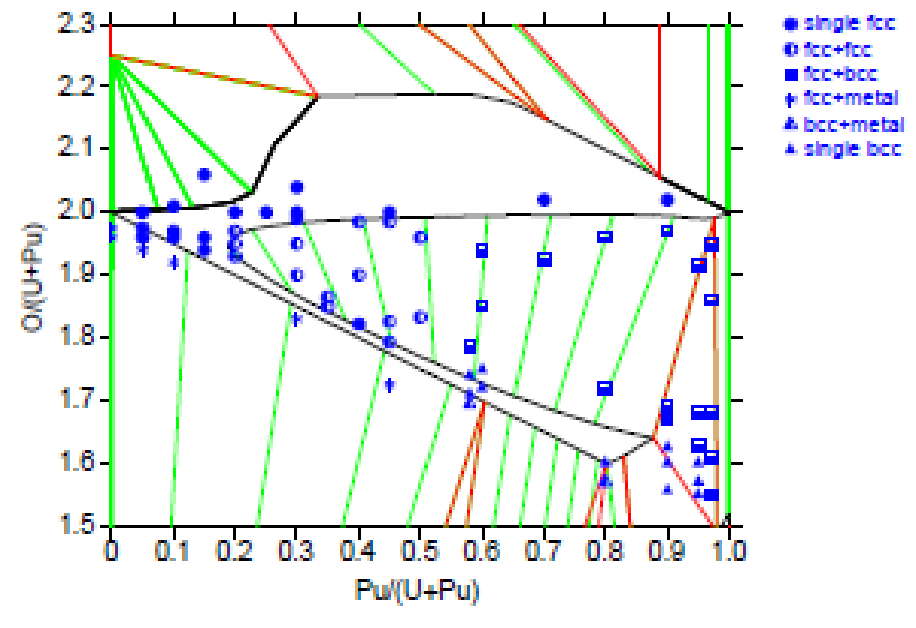

Fig. 2 : U-Pu-O phase diagram at $673^{\circ} \mathrm{K}$ showing the miscibility gap in the $\mathrm{MO}_{2}$ phase together with experimental data. 


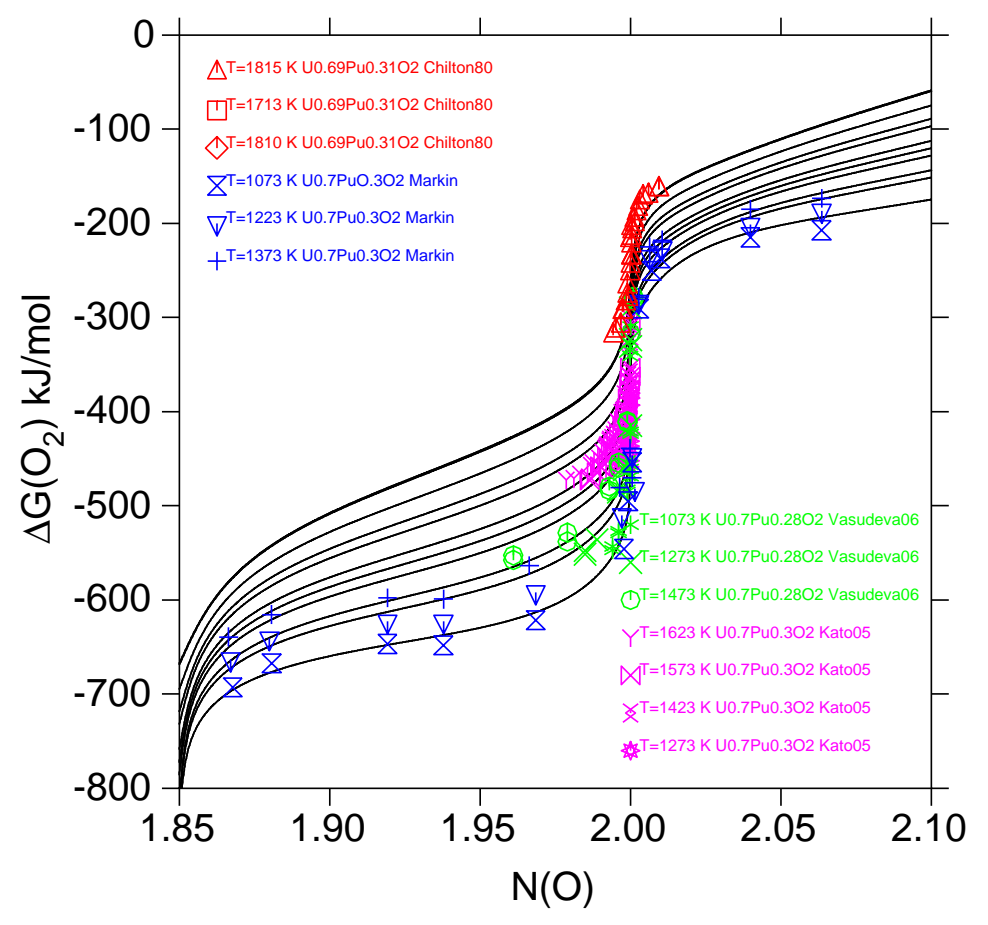

Fig. 3 : Oxygen chemical potentials at different temperatures and varying oxygen content for systems with Pu/u ratios around 0.3, together with experimantal data.

\section{References}

[1] H. Lukas, S. Fries, B. Sundman, Computational Thermodynamics. Cambridge University Press (2007).

[2] C. Guéneau, N. Dupin, B. Sundman, C. Martial, J.-C. Dumas, S. Gossé, S. Chatain, F. De Bruycker, D. Manar, R.J.M. Konings, Thermodynamic modeling of advanced oxide and carbide nuclear fuels: description of the U-Pu-O-C systems. J. Nucl Mater. 419 (2011) 145 


\title{
The use of Computational Thermodynamics to predict properties of multicomponent materials for nuclear applications
}

\author{
Bo Sundman and Christine Guéneau
}

INSTN and DEN/DANS/DPC/SCCME, CEA Saclay

December 5, 2012 


\section{Abstract}

Computational Thermodynamics is based on physically realistic models to describe metallic and oxide crystalline phases as well as the liquid and gas in a consistent manner. The models are used to assess experimental and theoretical data for many different materials and several thermodynamic databases has been developed for steels, ceramics, semiconductor materials as well as materials for nuclear applications.

Within CEA a long term work is ongoing to develop a database for the properties of nuclear fuels and structural materials.

An overview of the modelling technique will be given and several examples of the application of the database to different problems. 


\section{Outline}

\section{- Thermodynamics}

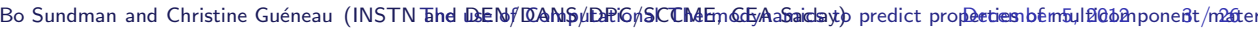




\section{Outline}

- Thermodynamics

- Computational Thermodynamics (CT, Calphad)

- models. 


\section{Outline}

- Thermodynamics

- Computational Thermodynamics (CT, Calphad)

- models,

- software 


\section{Outline}

- Thermodynamics

- Computational Thermodynamics (CT, Calphad)

- models,

- software

- assessments and databases 


\section{Outline}

- Thermodynamics

- Computational Thermodynamics (CT, Calphad)

- models,

- software

- assessments and databases

- The fuelbase database. 


\section{Outline}

- Thermodynamics

- Computational Thermodynamics (CT, Calphad)

- models,

- software

- assessments and databases

- The fuelbase database.

- Modelling oxides. 


\section{Outline}

- Thermodynamics

- Computational Thermodynamics (CT, Calphad)

- models,

- software

- assessments and databases

- The fuelbase database.

- Modelling oxides.

- Summary and conclusions 


\section{Outline}

- Thermodynamics

- Computational Thermodynamics (CT, Calphad)

- models,

- software

- assessments and databases

- The fuelbase database.

- Modelling oxides.

- Summary and conclusions

Literature:

- Nigel Saunders and Peter Miodownik, Calphad, Pergamon Materials Series, Vol 1 (1998)

- Mats Hillert: Phase Equilibria, Phase Diagrams and Phase Transformations, 2nd edition, Cambridge (2008).

- Leo Lukas, Suzana Fries and Bo Sundman, Computational Thermodynamics, Cambridge (2007). 


\section{Classical Thermodynamics}

Thermodynamics is a phenomenological theory describing the relation between some observable properties like temperature, $T$, pressure, $P$, heat $Q$, etc. 


\section{Classical Thermodynamics}

Thermodynamics is a phenomenological theory describing the relation between some observable properties like temperature, $T$, pressure, $P$, heat $Q$, etc.

It is founded on two simple laws for macroscopic systems:

1. Energy cannot be destroyed or created, 


\section{Classical Thermodynamics}

Thermodynamics is a phenomenological theory describing the relation between some observable properties like temperature, $T$, pressure, $P$, heat $Q$, etc.

It is founded on two simple laws for macroscopic systems:

1. Energy cannot be destroyed or created,

2. Heat never flows spontaneously from a cold body to a hot body 


\section{Classical Thermodynamics}

Thermodynamics is a phenomenological theory describing the relation between some observable properties like temperature, $T$, pressure, $P$, heat $Q$, etc.

It is founded on two simple laws for macroscopic systems:

1. Energy cannot be destroyed or created,

2. Heat never flows spontaneously from a cold body to a hot body

These laws, and some trivial mathematics, makes it possible to define a number of additional properties like internal energy, $U$, entropy, $S$, Gibbs energy, $G$ etc. These are not observables but can be used to derive strict mathematical relations between many properties. 


\section{Properties derived from the Gibbs energy}

$$
\begin{aligned}
S & =-\left(\frac{\partial G}{\partial T}\right)_{P, N_{i}} \\
H & =G-T S \\
V & =\left(\frac{\partial G}{\partial P}\right)_{T, N_{i}} \\
\mu_{i} & =\left(\frac{\partial G}{\partial N_{i}}\right)_{T, P, N_{j \neq i}}
\end{aligned}
$$

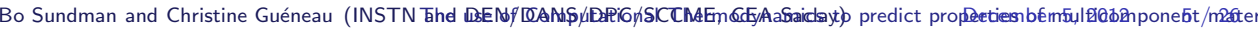




\section{Properties derived from the Gibbs energy}

$$
\begin{aligned}
S & =-\left(\frac{\partial G}{\partial T}\right)_{P, N_{i}} \\
H & =G-T S \\
V & =\left(\frac{\partial G}{\partial P}\right)_{T, N_{i}} \\
\mu_{i} & =\left(\frac{\partial G}{\partial N_{i}}\right)_{T, P, N_{j \neq i}} \\
C_{P} & =-T\left(\frac{\partial^{2} G}{\partial T^{2}}\right)_{P, N_{i}} \\
\alpha & =\frac{1}{V}\left(\frac{\partial^{2} G}{\partial P \partial T}\right)_{N_{i}} \\
\kappa & =-\frac{1}{V}\left(\frac{\partial^{2} G}{\partial P^{2}}\right)_{T, N_{i}}
\end{aligned}
$$

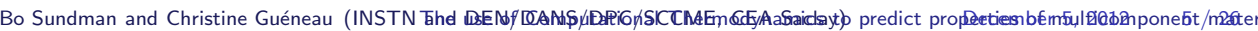




\section{Computational Thermodynamics: Central part of science}

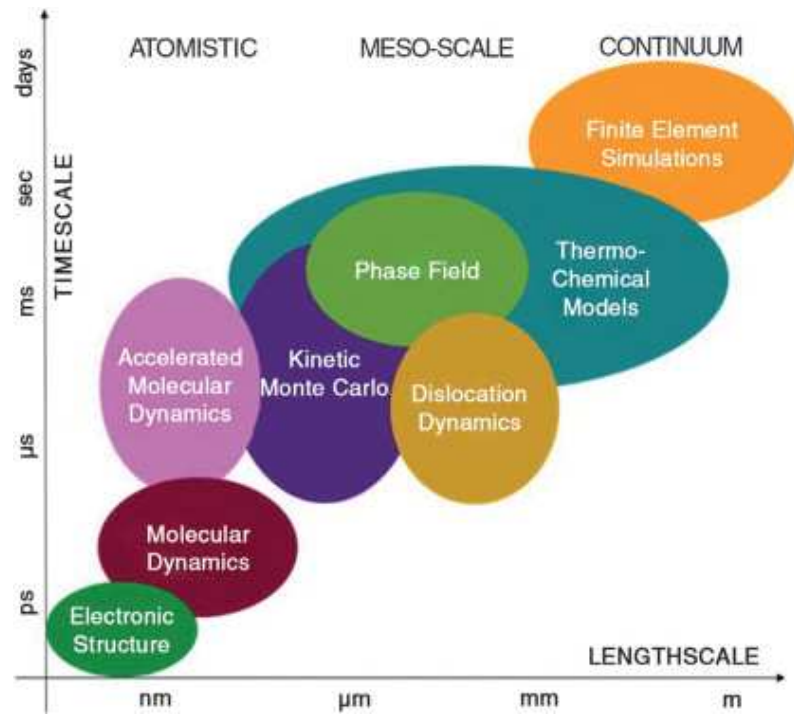

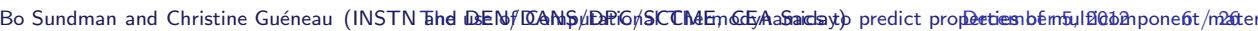




\section{Computational Thermodynamics: Models 1}

The Gibbs energy has been selected for modelling materials properties using the Calphad method. The main reason is that most experimental data is known at constant $T$ and $P$. 


\section{Computational Thermodynamics: Models 1}

The Gibbs energy has been selected for modelling materials properties using the Calphad method. The main reason is that most experimental data is known at constant $T$ and $P$.

$T$ and $P$ are intensive properties and simple polynomials can be used to describe the $T$ and $P$ dependence, but for very high pressures special models are needed. 


\section{Computational Thermodynamics: Models 1}

The Gibbs energy has been selected for modelling materials properties using the Calphad method. The main reason is that most experimental data is known at constant $T$ and $P$.

$T$ and $P$ are intensive properties and simple polynomials can be used to describe the $T$ and $P$ dependence, but for very high pressures special models are needed.

Modelling the composition dependence is the more complicated. There are two reasons for this:

- the amount of a component, $N_{i}$ is an extensive property, 


\section{Computational Thermodynamics: Models 1}

The Gibbs energy has been selected for modelling materials properties using the Calphad method. The main reason is that most experimental data is known at constant $T$ and $P$.

$T$ and $P$ are intensive properties and simple polynomials can be used to describe the $T$ and $P$ dependence, but for very high pressures special models are needed.

Modelling the composition dependence is the more complicated. There are two reasons for this:

- the amount of a component, $N_{i}$ is an extensive property,

- the configurational entropy is very important. 


\section{Computational Thermodynamics: Models 1}

The Gibbs energy has been selected for modelling materials properties using the Calphad method. The main reason is that most experimental data is known at constant $T$ and $P$.

$T$ and $P$ are intensive properties and simple polynomials can be used to describe the $T$ and $P$ dependence, but for very high pressures special models are needed.

Modelling the composition dependence is the more complicated. There are two reasons for this:

- the amount of a component, $N_{i}$ is an extensive property,

- the configurational entropy is very important.

For the configurational entropy one must take into account the formation of molecules in a gas phase, crystalline sites in solids, charge transfer between elements, clusters etc. In many cases it is necessary to introduce more constituents of the phases than just the components. 


\section{Computational Thermodynamics: Models 2}

Most materials consists of several crystalline phases with different structure and properties. The material often interact with other phases like gas and liquids.

The Gibbs energy is an extensive property and it is possible to model each phase separately:

$$
G=\sum_{\alpha} \aleph^{\alpha} G_{m}^{\alpha}\left(T, P, y_{i}\right)
$$

where $\aleph^{\alpha}$ is the number of moles and $G_{m}^{\alpha}$ is the molar Gibbs energy of of the phase $\alpha$. 


\section{Computational Thermodynamics: Models 2}

Most materials consists of several crystalline phases with different structure and properties. The material often interact with other phases like gas and liquids.

The Gibbs energy is an extensive property and it is possible to model each phase separately:

$$
G=\sum_{\alpha} \aleph^{\alpha} G_{m}^{\alpha}\left(T, P, y_{i}\right)
$$

where $\aleph^{\alpha}$ is the number of moles and $G_{m}^{\alpha}$ is the molar Gibbs energy of of the phase $\alpha$. The molar Gibbs energy is written as a function of the constituent fractions, $y_{i}$, to model the configuration of the phase. In this way each phase can be modelled independently. 


\section{Computational Thermodynamics: Models 2}

Most materials consists of several crystalline phases with different structure and properties. The material often interact with other phases like gas and liquids.

The Gibbs energy is an extensive property and it is possible to model each phase separately:

$$
G=\sum_{\alpha} \aleph^{\alpha} G_{m}^{\alpha}\left(T, P, y_{i}\right)
$$

where $\aleph^{\alpha}$ is the number of moles and $G_{m}^{\alpha}$ is the molar Gibbs energy of of the phase $\alpha$.The molar Gibbs energy is written as a function of the constituent fractions, $y_{i}$, to model the configuration of the phase. In this way each phase can be modelled independently.

The equilibrium is found by minimizing the total Gibbs energy for the given set of external conditions. 


\section{Computational Thermodynamics: Models 3}

In a gas phase the molecules like $\mathrm{H}_{2}, \mathrm{H}_{2} \mathrm{O}, \mathrm{O}_{2}$ etc. are the constituents. The mole fraction of component $i$ in the gas is

$$
x_{i}^{\text {gas }}=\frac{\sum_{j} b_{i j} y_{j}^{\text {gas }}}{\sum_{k} \sum_{j} b_{k j} y_{j}^{\text {gas }}}
$$

where $b_{i j}$ is the stoichiometric ratio of component $i$ in $j$. 


\section{Computational Thermodynamics: Models 3}

In a gas phase the molecules like $\mathrm{H}_{2}, \mathrm{H}_{2} \mathrm{O}, \mathrm{O}_{2}$ etc. are the constituents. The mole fraction of component $i$ in the gas is

$$
x_{i}^{\text {gas }}=\frac{\sum_{j} b_{i j} y_{j}^{\text {gas }}}{\sum_{k} \sum_{j} b_{k j} y_{j}^{\text {gas }}}
$$

where $b_{i j}$ is the stoichiometric ratio of component $i$ in $j$. In a crystalline phase one may have several sublattices with are preferred by different elements.

$$
x_{i}^{\alpha}=\frac{\sum_{s} a_{s} \sum_{j} b_{i j} y_{j}^{(s), \alpha}}{\sum_{s} a_{s} \sum_{k} \sum_{j} b_{k j} y_{j}^{(s), \alpha}}
$$

where $a_{s}$ is the number of sites on sublattice $s, b_{i j}$ is the stoichiometric factor and $y_{i}^{(s)}$ is the fraction of constituent $i$ on sublattice $s$. 


\section{Computational Thermodynamics: Models 4}

The figures below represent three crystalline structures, $\mathrm{B} 1, \mathrm{D} 8_{b}$ and $\mathrm{DO}_{3}$ which require sublattices to be modelled.
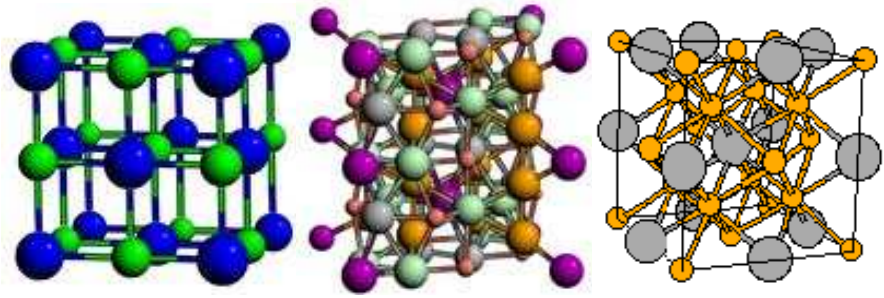

The Compound Energy Formalism (CEF) assumes random mixing of the constituents on each sublattice which gives the configurational entropy as

$$
{ }^{\mathrm{cfg}} S_{m}=\sum_{s} a_{s} \sum_{i} y_{i}^{(s)} \ln \left(y_{i}^{(s)}\right)
$$




\section{Computational Thermodynamics: Models 5}

The end member is an important concept in CEF defining one specific constituent in each sublattice. This defines a compound and the surface of reference for the phase:

$$
{ }^{\operatorname{srf}} G_{m}=\sum_{l} \prod_{i_{l}} y_{i}^{(s)}{ }^{\circ} G_{l}
$$

where $I$ has one constituent $i$ in each subalttice $s$ and ${ }^{\circ} G /$ is the Gibbs energy of formation of this compound from the reference states of the elements, depending only on $T$ and $P$. 


\section{Computational Thermodynamics: Models 5}

The end member is an important concept in CEF defining one specific constituent in each sublattice. This defines a compound and the surface of reference for the phase:

$$
{ }^{\mathrm{srf}} G_{m}=\sum_{l} \prod_{i_{l}} y_{i}^{(s) \circ} \mathrm{G}_{I}
$$

where $/$ has one constituent $i$ in each subalttice $s$ and ${ }^{\circ} G$ is the Gibbs energy of formation of this compound from the reference states of the elements, depending only on $T$ and $P$. In order to represent the interaction energy between the constituents in sublatticies there is an excess Gibbs energy:

$$
{ }^{E} G_{m}=\sum_{J} \prod_{j J} y_{j}^{(s)} L_{J}
$$

where $J$ has one or more constituents in each sublattice and $L_{J}$ describe the properties of real phases. 


\section{Computational Thermodynamics: Software 1}

There are several commercial software for equilibrium calculations and they offer slightly different ways to control a system. The simplest way is to specify $T, P$ and the amount of all components. 


\section{Computational Thermodynamics: Software 1}

There are several commercial software for equilibrium calculations and they offer slightly different ways to control a system. The simplest way is to specify $T, P$ and the amount of all components. But a user may prefer to specify the chemical potential, $\mu_{i}$ of a component $i$ or its activity or one or more of the stable phases or maybe even the composition of a specific phase. 


\section{Computational Thermodynamics: Software 1}

There are several commercial software for equilibrium calculations and they offer slightly different ways to control a system. The simplest way is to specify $T, P$ and the amount of all components. But a user may prefer to specify the chemical potential, $\mu_{i}$ of a component $i$ or its activity or one or more of the stable phases or maybe even the composition of a specific phase.

By varying one of the conditions one can calculate how the system varies with this and that is known as a property diagram.

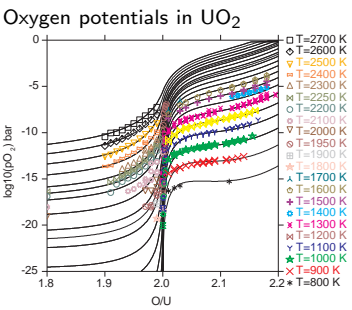




\section{Computational Thermodynamics: Software 1}

There are several commercial software for equilibrium calculations and they offer slightly different ways to control a system. The simplest way is to specify $T, P$ and the amount of all components.

But a user may prefer to specify the chemical potential, $\mu_{i}$ of a component $i$ or its activity or one or more of the stable phases or maybe even the composition of a specific phase.

By varying one of the conditions one can calculate how the system varies with this and that is known as a property diagram.
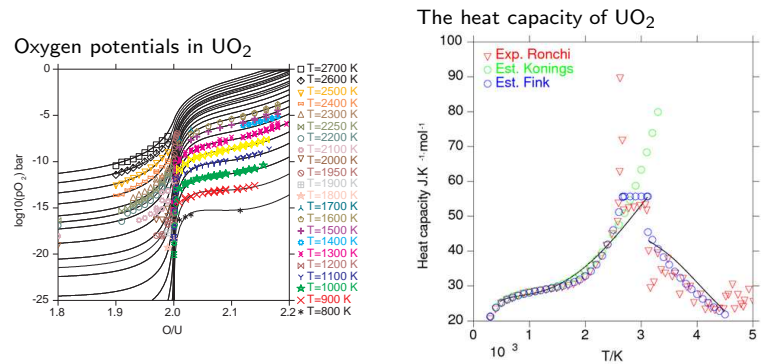

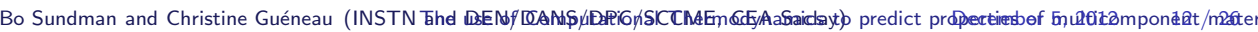




\section{Computational Thermodynamics: Software 1}

There are several commercial software for equilibrium calculations and they offer slightly different ways to control a system. The simplest way is to specify $T, P$ and the amount of all components.

But a user may prefer to specify the chemical potential, $\mu_{i}$ of a component $i$ or its activity or one or more of the stable phases or maybe even the composition of a specific phase.

By varying one of the conditions one can calculate how the system varies with this and that is known as a property diagram.
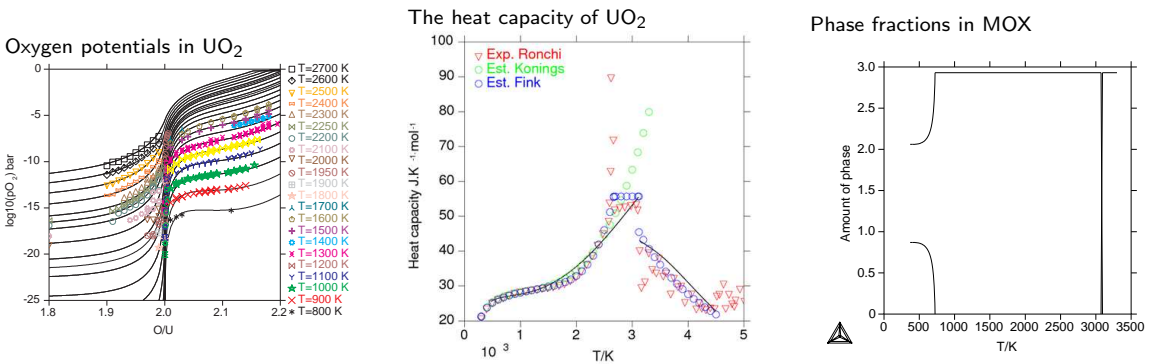

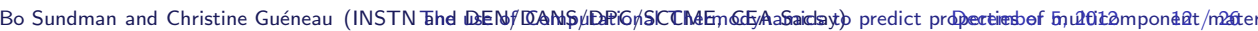




\section{Computational Thermodynamics: Software 2}

When two or more conditions are allowed to vary the software will calculate a phase diagram where the lines separate regions with different sets of stable phases.

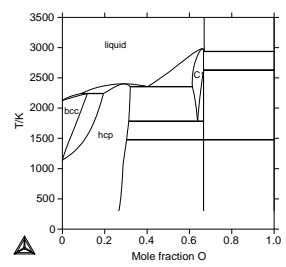

O-Zr phase diagram

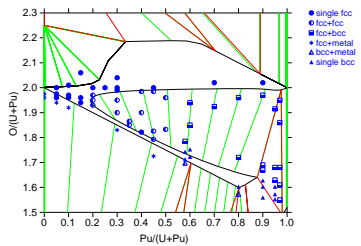

Isothermal section of O-Pu-U

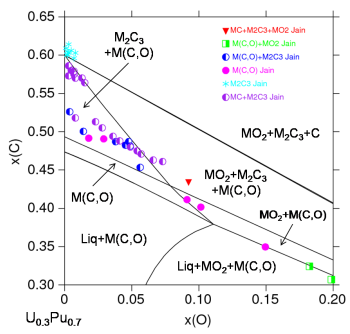

Isopleth of C-O-Pu-U

The leftmost diagram is the $\mathrm{O}-\mathrm{Zr}$ phase diagram, the middle diagram is an isothermal section at $473 \mathrm{~K}$ of the $\mathrm{O}-\mathrm{Pu}-\mathrm{U}$ phase diagram and the rightmost an isopleth section of the $\mathrm{C}-\mathrm{O}-\mathrm{Pu}-\mathrm{U}$ system. 


\section{Computational Thermodynamics: Software 3}

The diagrams below show the modelled Gibbs energy functions for two phases in a binary system at 3 different temperatures.
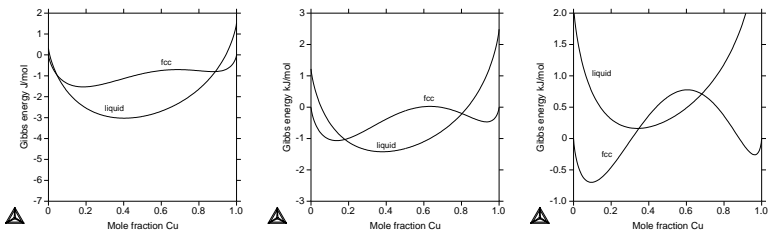


\section{Computational Thermodynamics: Software 3}

The diagrams below show the modelled Gibbs energy functions for two phases in a binary system at 3 different temperatures.
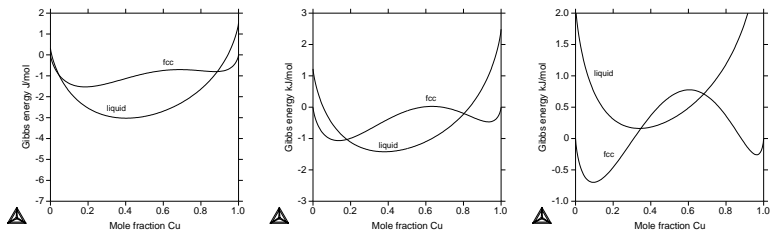

The equilibrium state for any temperature and composition is the lowest Gibbs energy. 


\section{Computational Thermodynamics: Software 3}

The diagrams below show the modelled Gibbs energy functions for two phases in a binary system at 3 different temperatures.
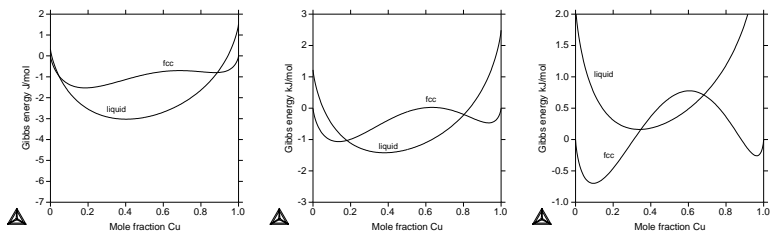

The equilibrium state for any temperature and composition is the lowest Gibbs energy. The end points to a tangent to a Gibbs energy curve gives the chemical potential of the components,

$$
\mu_{i}=\left(\frac{\partial G}{\partial N_{i}}\right)_{T, P, N_{j \neq i}}
$$




\section{Computational Thermodynamics: Software 3}

The diagrams below show the modelled Gibbs energy functions for two phases in a binary system at 3 different temperatures.
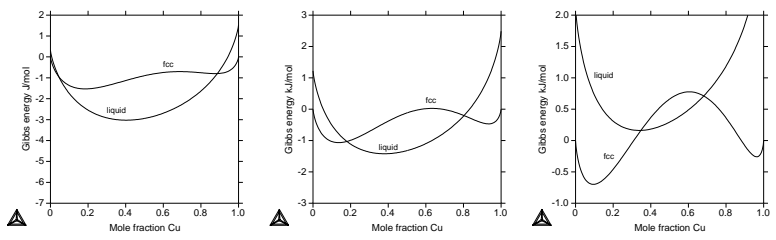

The equilibrium state for any temperature and composition is the lowest Gibbs energy. The end points to a tangent to a Gibbs energy curve gives the chemical potential of the components,

$$
\mu_{i}=\left(\frac{\partial G}{\partial N_{i}}\right)_{T, P, N_{j \neq i}}
$$

As the Gibbs energy curves are modelled outside the stable range of the phases it is possible to calculate metastable states. 


\section{Computational Thermodynamics: Software 3}

The diagrams below show the modelled Gibbs energy functions for two phases in a binary system at 3 different temperatures.
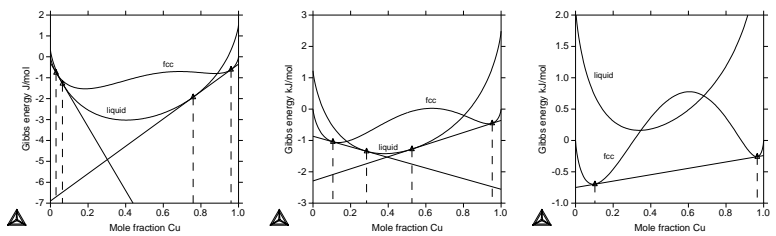

In two-phase regions the common tangents to the Gibbs energy curves gives the most stable state.

The vertical dashed lines indicate the compositions of the phases for the common tangents. 


\section{Computational Thermodynamics: Software 3}

The diagrams below show the modelled Gibbs energy functions for two phases in a binary system at 3 different temperatures.
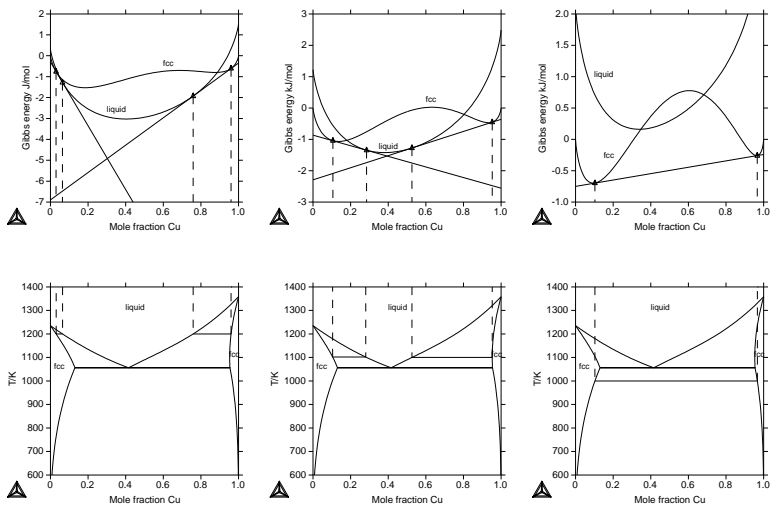

The solubility lines in the phase diagram are obtained by joining the points of the common tangents at varying temperatures.

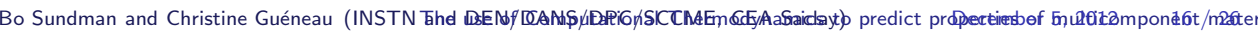




\section{Computational Thermodynamics: Databases 1}

In a CT database is stored:

- the model descriptions for each assessed phase, 


\section{Computational Thermodynamics: Databases 1}

In a CT database is stored:

- the model descriptions for each assessed phase,

- the end member energies, ${ }^{\circ} G_{l}$, 


\section{Computational Thermodynamics: Databases 1}

In a CT database is stored:

- the model descriptions for each assessed phase,

- the end member energies, ${ }^{\circ} G_{l}$,

- the interaction energies, $L_{J}$. 


\section{Computational Thermodynamics: Databases 1}

In a CT database is stored:

- the model descriptions for each assessed phase,

- the end member energies, ${ }^{\circ} G_{l}$,

- the interaction energies, $L_{J}$.

- additional data like magnetic parameters etc. 


\section{Computational Thermodynamics: Databases 1}

In a CT database is stored:

- the model descriptions for each assessed phase,

- the end member energies, ${ }^{\circ} G_{l}$,

- the interaction energies, $L_{J}$.

- additional data like magnetic parameters etc.

All these model parameters must be assessed, normally using experimental data, in binary and ternary systems. 


\section{Computational Thermodynamics: Databases 1}

In a CT database is stored:

- the model descriptions for each assessed phase,

- the end member energies, ${ }^{\circ} G_{l}$,

- the interaction energies, $L_{J}$.

- additional data like magnetic parameters etc.

All these model parameters must be assessed, normally using experimental data, in binary and ternary systems. Assessments are tideous and difficult tasks and requires great skill of the person doing the assessment, or his advisor. 


\section{Computational Thermodynamics: Databases 1}

In a CT database is stored:

- the model descriptions for each assessed phase,

- the end member energies, ${ }^{\circ} G_{l}$,

- the interaction energies, $L_{J}$.

- additional data like magnetic parameters etc.

All these model parameters must be assessed, normally using experimental data, in binary and ternary systems. Assessments are tideous and difficult tasks and requires great skill of the person doing the assessment, or his advisor. By combining several binary and ternary assessments one can construct multicomponent databases. Normally adjustments are needed to obtain correct multicomponent extrapolations. 


\section{Computational Thermodynamics: Databases 1}

In a CT database is stored:

- the model descriptions for each assessed phase,

- the end member energies, ${ }^{\circ} G_{l}$,

- the interaction energies, $L_{J}$.

- additional data like magnetic parameters etc.

All these model parameters must be assessed, normally using experimental data, in binary and ternary systems.

Assessments are tideous and difficult tasks and requires great skill of the person doing the assessment, or his advisor.

By combining several binary and ternary assessments one can construct multicomponent databases. Normally adjustments are needed to obtain correct multicomponent extrapolations.

Some model parameters, like the description of pure elements, must not be changed because that would make it impossible to combine assessments. 


\section{Computational Thermodynamics: Assessment 1}

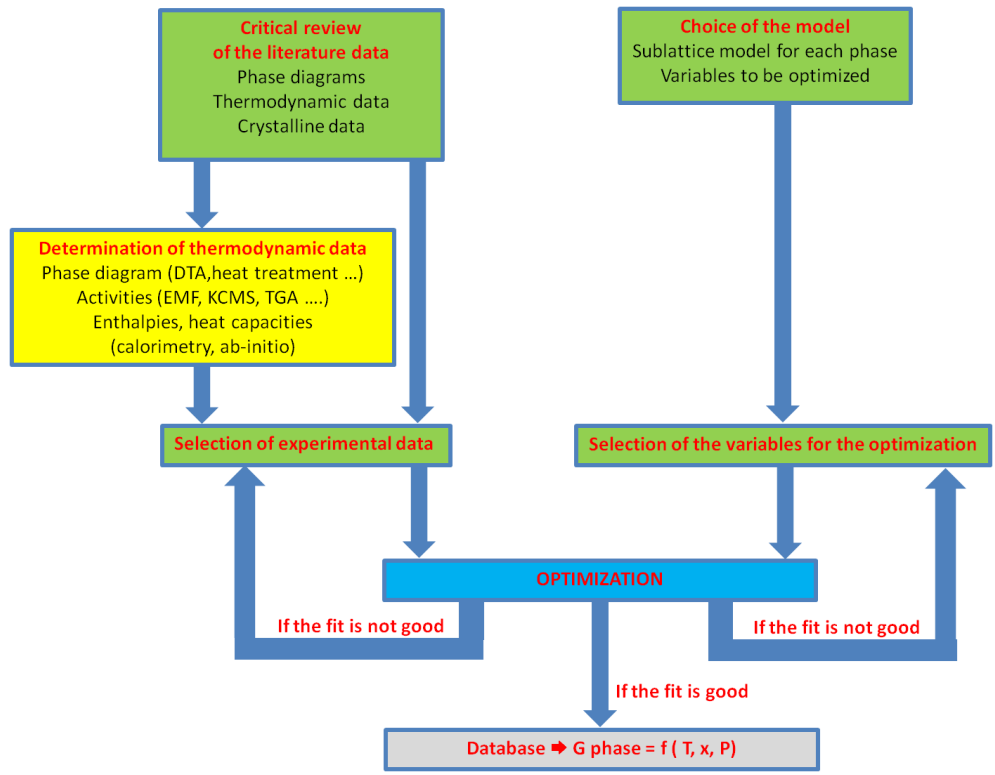

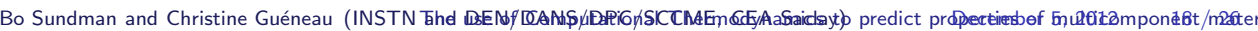




\section{Computational Thermodynamics: Assessment 2}

All kinds of data that can be calculated from the Gibbs energy of the system can, and must, be used to fit the model parameters.
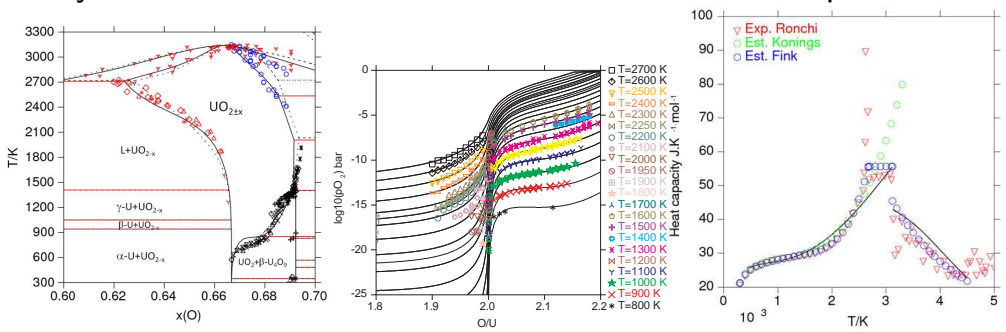

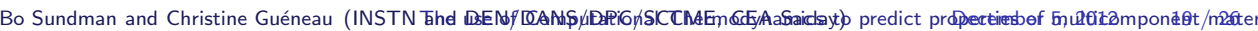




\section{Computational Thermodynamics: Assessment 2}

All kinds of data that can be calculated from the Gibbs energy of the system can, and must, be used to fit the model parameters.
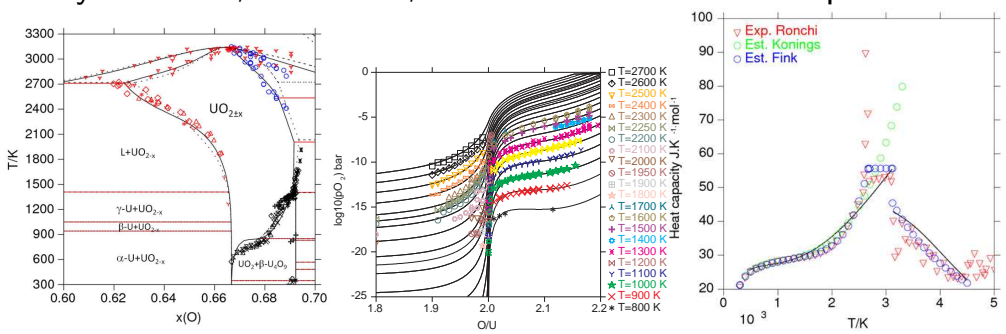

At CEA there is an ongoing project to develop the fuelbase database for nuclear fuels, fission products, and structural materials. 


\section{Computational Thermodynamics: Assessment 2}

All kinds of data that can be calculated from the Gibbs energy of the system can, and must, be used to fit the model parameters.
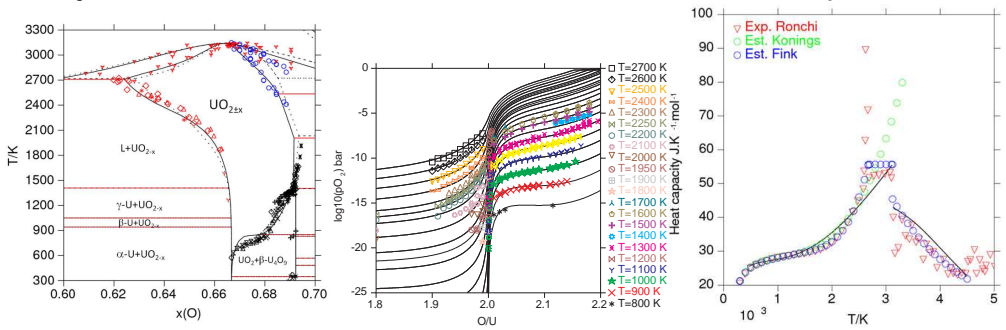

At CEA there is an ongoing project to develop the fuelbase database for nuclear fuels, fission products, and structural materials.

In recent assessments results from DFT calculations have been included for the formation of defects and metastable compounds. 


\section{Diagrams calculated from fuelbase}

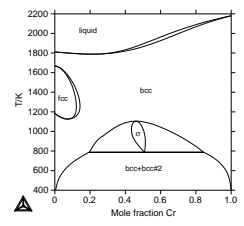

$\mathrm{Cr}-\mathrm{Fe}$

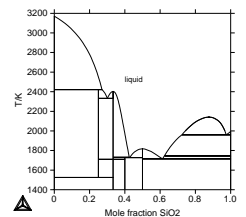

$\mathrm{CaO}-\mathrm{SiO}_{2}$

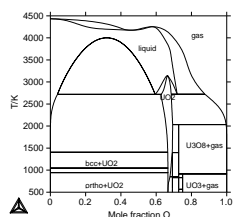

$\mathrm{O}-\mathrm{U}$

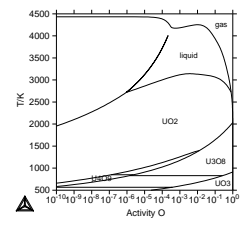

$\mathrm{O}-\mathrm{U}$

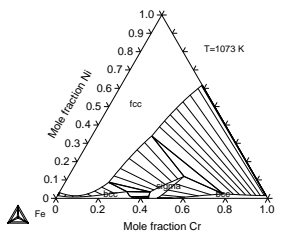

Cr-Fe-Ni at $1073 \mathrm{~K}$

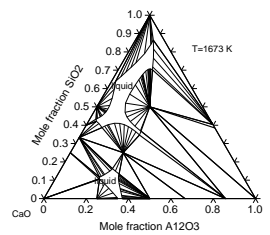

$\mathrm{Al}_{2} \mathrm{O}_{3}-\mathrm{CaO}-\mathrm{SiO}_{2}$ at $1673 \mathrm{~K}$

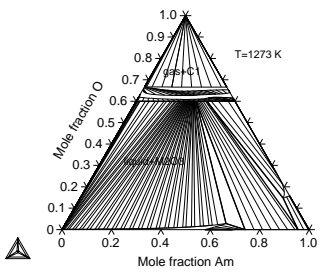

Isothermal section of Am-O-Pu at $1273 \mathrm{~K}$

Bo Sundman and Christine Guéneau (INSTN Thd WEENf שAAd\$ 


\section{Modelling ionic systems 1}

The difficulty with modelling oxides is the charge transfer. Normally each oxygen atom will take two electrons from the metallic atoms, some with multiple valencies, and a separate charge balance is needed for the equilibrium. 


\section{Modelling ionic systems 1}

The difficulty with modelling oxides is the charge transfer. Normally each oxygen atom will take two electrons from the metallic atoms, some with multiple valencies, and a separate charge balance is needed for the equilibrium. In crystalline phases vacancies are often needed to describe defects or deviations from stoichiometry.

A simple case is wustite (periclas, halite) with a B1 structure modelled as $\left(\mathrm{Fe}^{+2}, \mathrm{Fe}^{+3}, \mathrm{Va}\right)_{1}\left(\mathrm{O}^{-2}\right)_{1}$
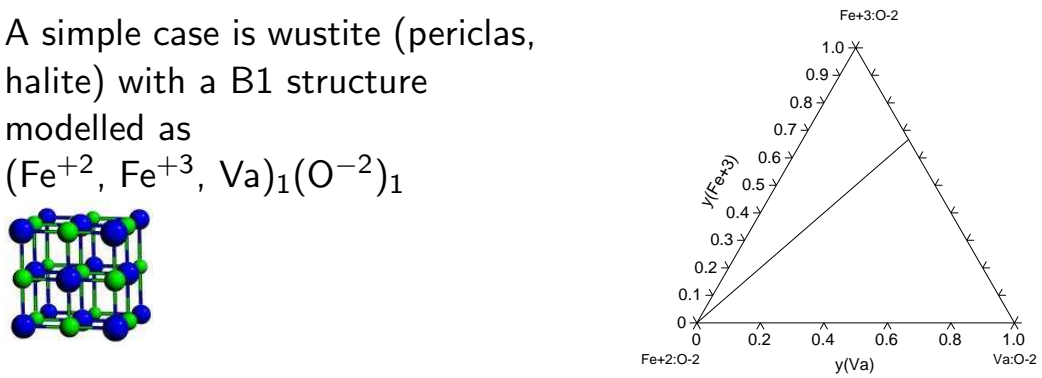


\section{Modelling ionic systems 2}

The $\mathrm{C} 1$ structure, $\mathrm{CaF}_{2}$, is the same structure as $\mathrm{MO}_{2}$ in nuclear fuels modelled with several metallic valencies and defects on the oxygen sublattice and interstitial oxygen. The shaded plane is the neutral combination of defects.

The end members can be drawn in different ways, either varying occupancy

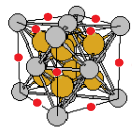
of the oxygen sublattices at constant valency of $U$ (top square prism) or varying $U$ valencies at constant occupancy of the oxygen sublattices (bottom triangular prism).
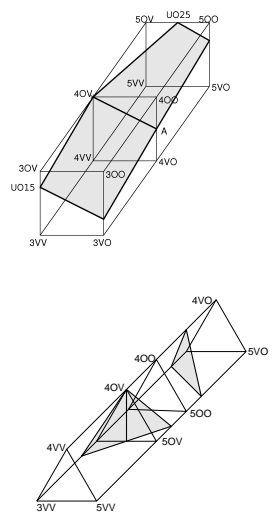


\section{Modelling ionic systems 2}

The $\mathrm{C} 1$ structure, $\mathrm{CaF}_{2}$, is the same structure as $\mathrm{MO}_{2}$ in nuclear fuels modelled with several metallic valencies and defects on the oxygen sublattice and interstitial oxygen. The shaded plane is the neutral combination of defects.

The end members can be drawn in different ways, either varying occupancy

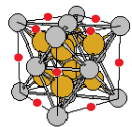
of the oxygen sublattices at constant valency of $U$ (top square prism) or varying $U$ valencies at constant occupancy of the oxygen sublattices (bottom triangular prism).

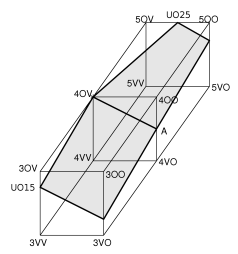

For the U-Pu-O system the model is:

$$
\left(\mathrm{U}^{+3}, \mathrm{U}^{+4}, \mathrm{U}^{+5}, \mathrm{Pu}^{+3}, \mathrm{Pu}^{+4}\right)_{1}\left(\mathrm{O}^{-2}, \mathrm{Va}\right)_{2}\left(\mathrm{O}^{-2}, \mathrm{Va}\right)_{1}
$$




\section{Modelling ionic systems 2}

Most of the end members of the $\mathrm{UO}_{2}$ model have a net charge and cannot be measured or even calculated by DFT. But one can make neutral combinations related to the formation of compounds.

The reaction for electronic defects is $2 \mathrm{U}^{+4}=\mathrm{U}^{+3}+\mathrm{U}^{+5}$

and expressed by the difference of 3 end members

${ }^{\circ} G_{3: \mathrm{O}: \mathrm{V}}+{ }^{\circ} \mathrm{G}_{5: \mathrm{O}: \mathrm{V}}-2{ }^{\circ} \mathrm{G}_{4: \mathrm{O}: \mathrm{V}}$

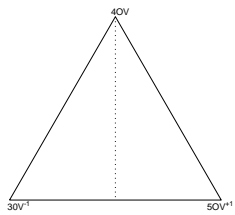




\section{Modelling ionic systems 2}

Most of the end members of the $\mathrm{UO}_{2}$ model have a net charge and cannot be measured or even calculated by DFT. But one can make neutral combinations related to the formation of compounds.

The reaction for electronic defects is $2 \mathrm{U}^{+4}=\mathrm{U}^{+3}+\mathrm{U}^{+5}$

and expressed by the difference of 3 end members

${ }^{\circ} G_{3: \mathrm{O}: \mathrm{V}}+{ }^{\circ} \mathrm{G}_{5: \mathrm{O}: \mathrm{V}}-2{ }^{\circ} \mathrm{G}_{4: \mathrm{O}: \mathrm{V}}$

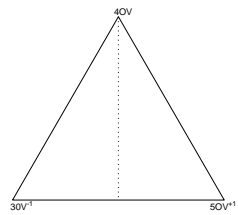

The Frenkel defects forming interstitial oxygen is given by

${ }^{\circ} G_{4: \mathrm{O}: \mathrm{O}}+{ }^{\circ} \mathrm{G}_{4: \mathrm{V}: \mathrm{O}}-2{ }^{\circ} \mathrm{G}_{4: \mathrm{O}: \mathrm{V}}$

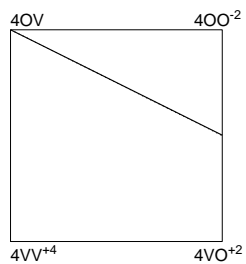




\section{Summary}

Calculations using a thermodynamic databases must be combined with other information and models in order to describe the real behaviour of a material. 


\section{Summary}

Calculations using a thermodynamic databases must be combined with other information and models in order to describe the real behaviour of a material.

- Thermodynamics models can take some defects into account, like vacancies, interstitials, anti-site atoms etc. 


\section{Summary}

Calculations using a thermodynamic databases must be combined with other information and models in order to describe the real behaviour of a material.

- Thermodynamics models can take some defects into account, like vacancies, interstitials, anti-site atoms etc.

- It is also possible to include kinetic data like mobilities in the databases but that require additional assessment work. 


\section{Summary}

Calculations using a thermodynamic databases must be combined with other information and models in order to describe the real behaviour of a material.

- Thermodynamics models can take some defects into account, like vacancies, interstitials, anti-site atoms etc.

- It is also possible to include kinetic data like mobilities in the databases but that require additional assessment work.

- The thermodynamic models can be extrapolated to metastable states and calculate the driving forces for preciptitation of new phases. 


\section{Summary}

Calculations using a thermodynamic databases must be combined with other information and models in order to describe the real behaviour of a material.

- Thermodynamics models can take some defects into account, like vacancies, interstitials, anti-site atoms etc.

- It is also possible to include kinetic data like mobilities in the databases but that require additional assessment work.

- The thermodynamic models can be extrapolated to metastable states and calculate the driving forces for preciptitation of new phases.

- Thermodynamics provide information on the gradients in chemical potential driving the diffusion in phase transformations. 


\section{Summary}

Calculations using a thermodynamic databases must be combined with other information and models in order to describe the real behaviour of a material.

- Thermodynamics models can take some defects into account, like vacancies, interstitials, anti-site atoms etc.

- It is also possible to include kinetic data like mobilities in the databases but that require additional assessment work.

- The thermodynamic models can be extrapolated to metastable states and calculate the driving forces for preciptitation of new phases.

- Thermodynamics provide information on the gradients in chemical potential driving the diffusion in phase transformations.

- The thermodynamic factor, the matrix with the second derivatives of the Gibbs energy, is needed to evaluate diffusion coefficients. 


\section{Conclusions}

There are many factors which must be modelled and determined experimentally in order to understand the behaviour of multicomponent materials. 


\section{Conclusions}

There are many factors which must be modelled and determined experimentally in order to understand the behaviour of multicomponent materials.

With an assessed thermodynamic database it is easy to calculate the set of stable set of phases, their amount and composition and the chemical potentials of the components for the varying external conditions. 


\section{Conclusions}

There are many factors which must be modelled and determined experimentally in order to understand the behaviour of multicomponent materials.

With an assessed thermodynamic database it is easy to calculate the set of stable set of phases, their amount and composition and the chemical potentials of the components for the varying external conditions.

This is a great help to select critical experimental work and to use in software for simulation of phase transformations. 


\title{
Thanks
}

\author{
for listening
}

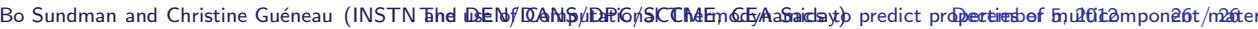

